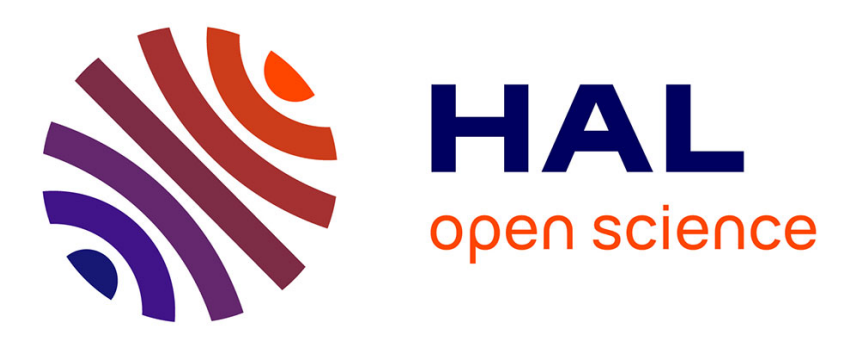

\title{
Cyclopropenyl Cation - the Simplest Huckel's Aromatic Molecule - and its Cyclic Methyl Derivatives in Titan's Upper Atmosphere.
}

\author{
Arshad Ali, Edward.C. Jr. Sittler, Dennis J. Chornay, Bertrand Rowe, \\ Cristina Puzzarini
}

\section{To cite this version:}

Arshad Ali, Edward.C. Jr. Sittler, Dennis J. Chornay, Bertrand Rowe, Cristina Puzzarini. Cyclopropenyl Cation - the Simplest Huckel's Aromatic Molecule - and its Cyclic Methyl Derivatives in Titan's Upper Atmosphere.. Planetary and Space Science, 2013, 87, pp.96-105. 10.1016/j.pss.2013.07.007 . hal-00916307

\section{HAL Id: hal-00916307 https://hal.science/hal-00916307}

Submitted on 10 Dec 2013

HAL is a multi-disciplinary open access archive for the deposit and dissemination of scientific research documents, whether they are published or not. The documents may come from teaching and research institutions in France or abroad, or from public or private research centers.
L'archive ouverte pluridisciplinaire HAL, est destinée au dépôt et à la diffusion de documents scientifiques de niveau recherche, publiés ou non, émanant des établissements d'enseignement et de recherche français ou étrangers, des laboratoires publics ou privés. 


\title{
Cyclopropenyl Cation - the Simplest Huckel's Aromatic Molecule - and its Cyclic Methyl Derivatives in Titan's Upper Atmosphere
}

\author{
A.Ali ${ }^{a, b,{ }^{*}}$, E.C.Sittler Jr. ${ }^{a}$, D.Chornay ${ }^{\mathrm{a}, \mathrm{b}}$, B.R.Rowe ${ }^{\mathrm{c}}$, C.Puzzarini ${ }^{\mathrm{d}^{*}}$ \\ ${ }^{a}$ NASA Goddard Space Flight Center, Greenbelt, Maryland 20771,USA \\ ${ }^{b}$ Department of Astronomy, University of Maryland, College Park, Maryland 20742, USA \\ 'Institut de Physique de Rennes, Equipe: Astrochimie Experimentale, CNRS, Universite de Rennes 1, \\ Campus de Beaulieu, 35042 Rennes Cedex, France \\ dDipartimento di Chimica “G.Ciamician” Universita di Bologna, via Selmi 2, 40126 Bologna, Italy \\ “Corresponding author: ashraf.ali@nasa.gov; cristina.puzzarini@unibo.it
}

\begin{abstract}
The recent measurements by Cassini Ion Neutral Mass Spectrometer (INMS) showed the presence of numerous carbocations and shed light on their composition in Titan's upper atmosphere. The present research identifies an important class of ion-molecule reactions proceeding via carbocation collision complexes, and its implications in the chemistry of Titan's thermosphere and ionosphere. An analysis (based on the kinetics and dynamics of the elementary chemical processes identified) of the Cassini measurements reveals the mechanism of formation of the three- membered Huckel aromatic ringsCyclopropenyl cation and its cyclic methyl derivatives. For carbocations, a nonclassical three-carboncenter two-electron-bond structure is no longer a controversial topic in chemistry literature. Emphasis has been placed on a future coordinated effort of state-of-the-art laboratory experiments, quantumchemical calculations, and astronomical ALMA and JWST observations including planetary in situ measurements at millimeter and submillimeter wavelengths to elucidate the structure, energetics and dynamics of the compositions of carbocations detected by Cassini cationic mass spectrometry. The cabocation chemistry in Titan's upper atmosphere has a possible bearing on the organic chemistry and aromaticity in the atmosphere of primitive earth.
\end{abstract}

\section{Introduction:}

Titan is the largest moon of Saturn and the second largest moon in our solar system. The recent measurements by instruments (Niemann et al., 2002; Waite et al., 2004; Young et al., 2004) onboard the Cassini spacecraft revealed that Titan's upper atmosphere harbors the richest atmospheric organic chemistry in the solar system (Vuitton et al., 2009a; Waite et al.,2007,2009; Coates et al., 2007; Vuitton 
et al., 2009b; Sittler et al., 2009; Ali et al., 2010). No other planets and their satellites in our solar system have been found to bear the level of chemical complexity that we observed on Titan by the CassiniHuygens space mission. The Cassini lon and Neutral Mass Spectrometer (INMS) detection (Waite et al., 2004) of composition of numerous carbocations (Vuitton et al., 2009a; Waite et al., 2007, 2009) and the detection of heavy negative ions (up to $13,800 \mathrm{amu} / \mathrm{q}$ ), in particular very large molecular anions (carbanions), by the Cassini CAPS Electron Spectrometer (Coates et al., 2007,2009) in Titan's thermosphere and ionosphere were two of the highly unexpected findings. Coates et al. (2009) also found a preference for larger negative ion mass at lower altitude.

Recent studies (Michael et al., 2011) suggest that these larger ions detected (Coates et al., 2007, 2009) are singly charged, and not super nucleophiles (i.e.,doubly charged negative ions). Michael et al. (2011) furthermore suggest that molecular charge may play a role in forming larger ions. The larger ions in Titan's ionosphere are labeled as "macromolecular" aerosol by Lavvas et al. (2013) rather than by the familiar term aerosol. These authors (Lavvas et al., 2013) showed that the mechanism of formation of macromolecules and their growth is directly related to the ion-neutral chemistry. However, the mechanisms involved in their formation remain largely unclear (Lavvas et al., 2013; Biennier et al., 2011). A better understanding of the mechanism of formation and growth of macromolecules and their compositions will require future studies of the molecular structure for the individual carbocations and carbanions detected by Cassini instruments and of the kinetics of ion-neutral reactions.

In this paper we will focus on the key issue of the chemistry of carbocations (Olah, 1972, 1995) in the gas phase in space, because their role is now well documented in the reactivity of both saturated hydrocarbons with single bonds and unsaturated hydrocarbons in many electrophilic reactions in synthetic organic chemistry. An understanding of the reactions of carbocation chemistry in the gas phase may shed light on the path toward prebiological molecules-organic inventory in the atmosphere on Titan.

Among the INMS detected cations, $\mathrm{CH}_{5}{ }^{+}$is the simplest Olah's carbocations (Olah, 1972, 1995). Is the carbocation chemistry in Titan's upper atmosphere largely governed by Olah's "nonclassical" carbonium ions (see below) based structure? The primary theme of "classical" structure of carbo-ions (where the general concepts of two electron two center (2e-2c) Lewis valence bonds are maintained) versus its "nonclassical" structure having electron-pair sharing a three-center bond formation was a great controversial issue, and largely debated some decades earlier. The reader is referred to H. C. Brown's monograph, 1977. A series of electrophilic ion-neutral fast reactions as shown below dominate Titan's thermosphere and ionosphere. These reactions proceed through carbocation collision complexes over the potential energy surfaces. The three-center bound nonclassical carbonium ion may lead to the classical trivalent carbenium global minimum via bond rearrangement. We might therefore seek at a clear differentiation of classical from nonclassial molecular structures through rotational and rotationvibration spectra of carbo-ions. This will bring a major advance in our future understanding of dynamics and reactivity of carbocations (Vuitton et al., 2009a) detected by Cassini cationic mass spectrometry. 
The most recent INMS mass spectrometric detection (Vuitton et al.,2009a; Waite et al., 2009) of protonated methane, $\mathrm{CH}_{5}{ }^{+}$, in Titan's upper atmosphere is highly intriguing. Its predicted (Muller et al., 1997; Marx et al., 1997; Schreiner et al., 1993) nonequivalent three equilibrium structures with almost identical energies and its five protons scrambling bring the question of feasibility of observation of hypercoordinated carbon in the laboratory (White et al., 1999; Boo et al., 1995; Asvany et al., 2005) and assigning it to a traditional global minimum molecular structure. Despite a great deal of sophisticated quantum chemical studies (Muller et al., 1997; Marx et al., 1997; Schreiner et al., 1993; Hinkle et al., 2011; Witt et al., 2011; Bowman et al., 2010) aimed at understanding of its chemical bonding, and a number of highly impressive experiments (White et al., 1999; Boo et al., 1995; Asvany et al., 2005; Huang et al., 2006; Ivanov et al., 2010; and references therein) for obtaining the infrared spectrum for $\mathrm{CH}_{5}{ }^{+}$, there is not yet a final resolution of its structure and dynamics. Moreover, astronomical observation of this species in space will undoubtedly require the knowledge of its rotational spectrum (Bunker et al., 2004). However, the very large amplitude internal motion of this ion will make it exceedingly difficult to obtain an accurate $\mathrm{mm}$-wave spectrum measured by experiment. One solution to this problem is perhaps to freeze out the scrambling and internal motions by bringing most of the populations of this ion to the rotational ground state at very low temperatures under cold and ultra-cold conditions. As a matter of fact, experimental techniques such as the pulsed CRESU technique (Canosa et al., 2008) are available to cool complex carbocations such as $\mathrm{CH}_{5}{ }^{+}$down to $1 \mathrm{~K}$ or even below. A complete understanding of molecular structure and dynamics is though difficult, but certainly feasible if one attempts to detect the $J=1 \leftarrow 0 \mathrm{~mm}$-wave absorption transition of isolated $\mathrm{CH}_{5}{ }^{+}$ion in a radiofrequency (rf) trap at a temperature close to $1 \mathrm{~K}$ or further lower (Bunker et al., 2004; Gerlich, 2005)

Furthermore, the discover of protonated forms of acetylene, methylacetylene, and dimethylacetylene in Titan's upper atmosphere prompt the question whether Olah's " nonclassical" structures of carbo-ions are feasible to observe in space and in the laboratory in the gas phase (Saykally, 1988). The longstanding pioneering laboratory infrared spectroscopic measurements of Oka 's research group for protonated acetylene clearly establishes that the nonclassical bridged structure of $\mathrm{C}_{2} \mathrm{H}_{3}{ }^{+}$is energetically the most stable (Crofton et al., 1989). The systematic splittings with the intensity ratio of 2:1 in some parts of the very rich vibration-rotation spectrum in the $3.2 \mu \mathrm{m}$ region of the antisymmetric $\mathrm{C}-\mathrm{H}$ stretching $\left(v_{6}\right.$ band) have been ascribed to tunneling splittings (Crofton et al., 1989). These tunneling splittings are due to the apex proton and the two end protons in the bridged structure, which exchange their positions with a measurable time scale. The primary theme of this infrared spectroscopic observation is that the nonclassical structure is lower in energy than the classical structure, and the classical structure is rather a transition state between two equivalent forms of the nonclassical isomers (Crofton et al., 1989). The transition state barrier height is estimated to be $3.7 \pm 1.3 \mathrm{kcal} / \mathrm{mol}$ (Lindh et al., 1991). Concerning the internal motion of protons in this bridged structure of $\mathrm{C}_{2} \mathrm{H}_{3}{ }^{+}$, laboratory measurements of the millimeter and submillimeter wave spectrum of this carbo-ion have been carried out (Bogey et al., 1992), but there is no indication yet of the observation of tunneling splitting in rotational transitions in the ground vibrational state. 
The questions raised above are also applicable to many other carbocation systems such as protonated methylacetylene $\mathrm{C}_{3} \mathrm{H}_{5}{ }^{+}$and protonated dimethylacetylene $\mathrm{C}_{4} \mathrm{H}_{7}{ }^{+}$. As an example, in analogy to protonated acetylene, protonated methylacetylene $\mathrm{C}_{3} \mathrm{H}_{5}{ }^{+}$may have nonclassical two-electron threecenter bound bridged structure in addition to its classical counterparts such as the stable 2-methylvinyl cation. Will the apex proton and the electrophile $\mathrm{CH}_{3}{ }^{+}$in this bridged structure exchange their positions within a measurable time scale? In other words the question is whether a nonequivalent equilibrium carbonium isomer structure will also result where the donation of two electrons from the $\pi$ bond in acetylene into the vacant orbital of the apex electrophile $\mathrm{CH}_{3}{ }^{+}$are delocalized over three centers. The radioastronomical detection of floppy molecular structures of carbocations in space will require laboratory measurements of the microwave absorption spectrum of rotational transitions. Once again, the detection of ultracold ions in rf traps offers a viable route. The observation of nonclassical "floppy"structures in space is most likely feasible provided that we succeed in laboratory measurements in bringing most of the populations of these ions in their lowest values of $\mathrm{J}$ and $\mathrm{K}$.

The primary focus of this paper is the carbocation chemistry in Titan's upper atmosphere and the simplest Huckel Aromatic structure formation thereof. This includes the chemistry of a series of electrophilic ion-neutral reactions (see below) in Titan's organic chemistry where $\pi$-electron pair from unsaturated hydrocarbons is donated to the electrophile in the three-center bond. The $\sigma$-donation from saturated hydrocarbons also plays a critical role in forming (2e-3c) nonclassical carbonium structures. The saturated organic species have in fact the same abilities to share an electron pair from a single bond in forming delocalized three-center bond formation. The INMS observed both protonated methane $\mathrm{CH}_{5}{ }^{+}$ and protonated ethane $\mathrm{C}_{2} \mathrm{H}_{7}{ }^{+}$, clearly supporting this view.

Decades ago, Hoffmann (1964) applied the extended Huckel theory for carbo-ions and predicted a significant charge delocalization in many classical ion geometries, i.e., the nonclassical carbonium ion character is preserved in many carbocations. Thus, both classical and nonclassical features will contribute to the reactivity and chemistry of carbocation in Titan's upper atmosphere.

Temperatures, densities, and time scales are the three most important parameters that govern the gas phase reactions involving carbocations and carbanions in Titan's cold upper atmosphere. The organic chemistry is also driven by fast neutral reactions (Klippenstein and Georgievskii, 2008; Canosa et al., 2008) such as radical-molecule reactions. Since the translational energy of molecular species at temperatures of Titan's thermosphere and ionosphere is typically of the order of $1 \mathrm{~kJ} \mathrm{~mol}^{-1}$, only exothermic reactions with practically no entrance barriers can take place. Even reactions with slight endothermicity may not occur. Because of low densities, ternary reactions involving three-body collisions are mostly ineffective. In an elementary reaction the cold and ultracold temperatures prevail over the electronic and atomic dynamics in a chemical rearrangement process. Thus, the influence of reagent rotational states in molecular dynamics and reactivity of carbocations is of primary concern.

$\mathrm{Ab}$ initio quantum chemical predictions and measurements of rotational and rotation-vibration spectra at very low temperatures are the basic information required in the astronomical Atacama Large Millimeter Array (ALMA) interferometric observations of the molecular carbocations detected by INMS 
as well as in future observations from a submillimeter-wave heterodyne sounder onboard a Titan orbiter (Lellouch et al., 2010), and the James Webb Space Telescope (JWST) observations (Gardner et al., 2006). We note that these spectroscopic observations (see the discussion following) are vital for deriving molecular structural information on the composition of Titan's thermosphere and ionosphere revealed by the Cassini mass spectrometric instruments ( Vuitton et al., 2009a; Waite et al., 2009).

The $\mathrm{H}_{3}{ }^{+}$ion (Oka, 2006, 2012; Park and Light, 2007; Amano, 2006; Drossart et al., 1989) is the simplest case with a two-electron three-center bond, and in this sense this ion is the root to the understanding of the chemistry of electron-deficient carbonium structures (Longuet-Higgins, 1957). The laboratory infrared spectrum of this ion (Oka, 2006) including millimeter and sub-millimeter observation of its deuterium-containing isotopologues (Amano, 2006), and its first detection in nature in the infrared emission bands in the polar auroral regions of the Jupiter ionosphere (Drossart et al., 1989) have stimulated intensive searches that ended up with its observation in astrophysical plasma universe (Oka, $2006,2012)$. This ionic species plays a crucial role in the chemical evolution of nearby interstellar molecular clouds, the birthplace of stars and planetary systems (Herbst, 2005). Would the carbonium ion chemistry with similar chemical bonding characteristics in Titan's thermosphere and ionosphere be regarded as footprints for organic molecules in the atmosphere of extrasolar planets and young planetary systems around other stars? It is well acknowledged (Raulin et al., 2009) that organic chemistry in Titan's atmosphere is intimately linked to prebiotic organic synthesis in the atmosphere of primitive earth. The astronomical spectroscopic observations of organic molecules in the atmospheres of planets as their transit parent stars will most likely place a clear linkage between the chemistry in Titan's upper atmosphere and the organic chemistry and aromaticity (see below) in the atmosphere of young earth.

\section{Ion Neutral Mass Spectrometer (INMS) Observations and Analysis:}

Based on INMS Cassini observations (Vuitton et al., 2009a; Waite et al., 2004, 2009, 2007) of specific carbocations (Olah, 1972, 1995), such as $\mathrm{CH}_{3}{ }^{+}, \mathrm{CH}_{5}{ }^{+}, \mathrm{C}_{2} \mathrm{H}_{3}{ }^{+}, \mathrm{C}_{2} \mathrm{H}_{5}{ }^{+}, \mathrm{C}_{3} \mathrm{H}_{5}{ }^{+}$, and $\mathrm{C}_{3} \mathrm{H}_{7}{ }^{+}$, and on the analysis of previous laboratory crossed molecular beam measurements (Sonnenfroh and Farrar, 1986) and ab initio theoretical studies (Lopez et al., 1996; Almlof et al., 1984; Hariharan et al., 1974) of the reactions of $\mathrm{CH}_{3}{ }^{+}$ with ethylene and acetylene, in this paper we infer (see discussion below) that a significant fraction of the detected $\mathrm{C}_{3} \mathrm{H}_{3}{ }^{+}$composition (Vuitton et al., 2009a; Waite et al., 2009) by INMS in Titan's upper atmosphere is the stable Cyclopropenyl Cation - the simplest Huckel's Aromatic Molecule. What naturally follows is also the identification of an important class of reactions in Titan's upper atmosphere such as those of $\mathrm{CH}_{3}{ }^{+}$with methylacetylene and dimethylacetylene. The latter offers an explanation of the observed composition of $\mathrm{C}_{4} \mathrm{H}_{5}{ }^{+}$in terms of methyl substituted cyclopropenyl cation and of $\mathrm{C}_{5} \mathrm{H}_{7}{ }^{+}$ where two hydrogen atoms in the cyclopropenyl cation are substituted by two methyl groups (Vuitton et al., 2009a; Waite et al., 2007). 
In Titan's ionospheric chemistry, both $\mathrm{C}_{2} \mathrm{H}_{3}{ }^{+}$and $\mathrm{C}_{2} \mathrm{H}_{5}{ }^{+}$are the protonated forms of two abundant neutrals: acetylene and ethylene, respectively (Vuitton et al., 2009a; Waite et al., 2005, 2009). These ions are primarily formed by proton transfer reactions (Vuitton et al., 2009a) to the corresponding neutrals from ionic hydrocarbon species with smaller proton affinities. Below are shown four important ion neutral exothermic reaction channels (Sonnenfroh and Farrar, 1986; Lopez et al., 1996) that could be accessible at the low temperatures (i.e., from 10 to $200 \mathrm{~K}$ ) typical of interstellar clouds and planetary atmospheres:

$$
\begin{aligned}
& \mathrm{CH}_{3}^{+}+\mathrm{C}_{2} \mathrm{H}_{4} \rightarrow \mathrm{C}_{2} \mathrm{H}_{3}^{+}+\mathrm{CH}_{4} \quad \Delta \mathrm{H}=-1.02 \mathrm{eV}, \quad \text { (1) } \\
& \rightarrow \mathrm{C}_{3} \mathrm{H}_{5}^{+}+\mathrm{H} 2 \quad \Delta \mathrm{H}=-2.11 \mathrm{eV}, \quad \text { (2) } \\
& \rightarrow \mathrm{C}_{3} \mathrm{H}_{3}^{+}+2 \mathrm{H}_{2} \quad \Delta \mathrm{H}=-0.85 \mathrm{eV}, \quad \text { (3) } \\
& \mathrm{CH}_{3}^{+}+\mathrm{C}_{2} \mathrm{H}_{2} \rightarrow \mathrm{C}_{3} \mathrm{H}_{3}^{+}+\mathrm{H} 2, \quad \Delta \mathrm{H}=-2.65 \mathrm{eV},
\end{aligned}
$$

Both reactions presented above are known to involve condensation reactions proceeding through the formation of transient carbocation collision complexes. Ab initio (Lopez et al., 1996; Almlof et al., 1984; Hariharan et al., 1974) theoretical studies of the reaction paths and crossed beam measurements (Sonnenfroh and Farrar, 1986) of the products center of mass angular flux and translation energy distributions have been carried out to determine branching ratios of the product formation from the reaction of $\mathrm{CH}_{3}{ }^{+}$with ethylene, as well as the mechanism of formation of $\mathrm{C}_{3} \mathrm{H}_{3}{ }^{+}$from reaction (4) exclusively. We do note however that these crossed beam measurements (Sonnenfroh and Farrar, 1986) have been performed at collision energies between 0.5 and $2.0 \mathrm{eV}$. These initial energies are much higher than the average collision energy at the low temperatures prevailing in Titan's upper atmosphere.

The measurements of products center of mass angular flux distributions are usually indicative of the life times (a fraction of a rotational period) of short lived complexes participating in bimolecular reactions. Such studies have allowed (Sonnenfroh and Farrar, 1986) to construct a reaction pathway that leads to the initial formation of three -center two-electon bond (Olah, 1972, 1995; Longuet-Higgins, 1957) in metastable corner protonated cyclopropane $\left(\mathrm{C}_{3} \mathrm{H}_{7}{ }^{+}\right)$by an electrophilic attack of $\mathrm{CH}_{3}{ }^{+}$to the $\pi$ cloud of ethylene. Ab initio calculations of reation channels indicate (Sonnenfroh and Farrar, 1986; Almlof et al., 1984; Hariharan et al., 1974) that $\mathrm{C}_{3} \mathrm{H}_{7}^{+}$readily isomerizes by ring opening to the $n$-propyl cation, followed by a 1,2 hydrogen atom shift to the lowest energy sec- $\mathrm{C}_{3} \mathrm{H}_{7}{ }^{+}$structure. Decay (Sonnenfroh and Farrar, 1986) to $\mathrm{CH}_{4}+\mathrm{C}_{2} \mathrm{H}_{3}{ }^{+}$(hydride abstraction) from the sec- $\mathrm{C}_{3} \mathrm{H}_{7}{ }^{+}$minimum structure is endothermic by $60 \mathrm{kcal} \mathrm{mol}^{-1}$. In the literature no estimates are provided so far for what concerns the magnitude of the exit channel barrier for this process in reaction (1). The angular flux contour maps (Sonnenfroh and Farrar, 1986) for allyl cation $\left(\mathrm{C}_{3} \mathrm{H}_{5}{ }^{+}\right)$formation have some similarities to those for the $\mathrm{CH}_{4}$ reaction channel (1) and this suggests that both reaction channels (1) and (2) have a common precursor collision complex. Ab initio calculations show (Sonnenfroh and Farrar, 1986; Almlof et al., 1984) further that formation of the lowest energy allyl cation from the sec- $\mathrm{C}_{3} \mathrm{H}_{7}{ }^{+}$intermediate in reaction channel (2) proceeds over a significant exit barrier of approximately $51 \mathrm{kcal} \mathrm{mol}^{-1}$. This is consistent (Sonnenfroh and 
Farrar, 1986; Almlof et al., 1984) with the metastable mass spectrometric measurements (Burgers et al., 1983) of the kinetic energy release in the fragmentation of $\mathrm{C}_{3} \mathrm{H}_{7}{ }^{+}$and the reported product center of mass translational energy distribution (Sonnenfroh and Farrar, 1986) for $\mathrm{C}_{3} \mathrm{H}_{5}{ }^{+}$. In passing we do note the INMS detection of the composition of the stable allyl cation $\mathrm{C}_{3} \mathrm{H}_{5}{ }^{+}$.

The mechanistic interpretation of the cyclic- $\mathrm{C}_{3} \mathrm{H}_{3}{ }^{+}$isomer formation in the reaction channel (3) is drawn from the kinematics of crossed beam measurements (Sonnenfroh and Farrar, 1986). The reaction channel (3) above requires the loss of two molecules of hydrogen from the metastable adduct sec- $\mathrm{C}_{3} \mathrm{H}_{7}{ }^{+}$ formed from the colliding reagents. Is this process simultaneous or sequential? If the process of ejection of two molecules of hydrogen from the adduct occurs in a single event, the determination of the kinematics would be notoriously difficult, indeterminate. It is therefore assumed (Sonnenfroh and Farrar, 1986) that the initial complex $\mathrm{C}_{3} \mathrm{H}_{7}{ }^{+}$sequentially loses a single $\mathrm{H}_{2}$ molecule, and the resultant internally excited $\mathrm{C}_{3} \mathrm{H}_{5}{ }^{+}$forms $\mathrm{C}_{3} \mathrm{H}_{3}{ }^{+}$from the loss of another molecule of hydrogen in the reaction process (3). Because of the large difference in mass between $\mathrm{H}_{2}$ and the unobserved $\mathrm{C}_{3} \mathrm{H}_{5}{ }^{+}$in reaction channel (3), and since only highly internally excited $\mathrm{C}_{3} \mathrm{H}_{5}{ }^{+}$will dissociate to form $\mathrm{C}_{3} \mathrm{H}_{3}{ }^{+}$, it is reasonable to assume that the new center of mass velocity vector lies along the initial center of mass of the colliding reagents. In essence, the final hydrogen molecule is emitted relative to the original centroid of the collision system. This assumption allows (Sonnenfroh and Farrar, 1986) to extract from crossed beam measurements product center of mass angular flux and translational energy distributions for $\mathrm{C}_{3} \mathrm{H}_{3}{ }^{+}$ formation from the reaction of $\mathrm{CH}_{3}{ }^{+}$with $\mathrm{C}_{2} \mathrm{H}_{4}$ at various collision energies. The measurements (Sonnenfroh and Farrar, 1986) of angular flux distributions reflect that $\mathrm{C}_{3} \mathrm{H}_{3}{ }^{+}$formation proceeds by sequential ejection of two hydrogen molecules from the initial $\mathrm{C}_{3} \mathrm{H}_{7}^{+}$collision complex. It is important to note that the widths of the translational energy distribution (Sonnenfroh and Farrar, 1986) measurements for $\mathrm{C}_{3} \mathrm{H}_{3}{ }^{+}$formation require that a significant fraction of the $\mathrm{C}_{3} \mathrm{H}_{3}{ }^{+}$products must be the more stable cyclopropenium isomer. Ab initio studies (Lopez et al., 1996) show that the exit channel for the reaction of $\mathrm{CH}_{3}{ }^{+}$with acetylene to form $\mathrm{C}_{3} \mathrm{H}_{3}{ }^{+}$involves similar $\mathrm{C}_{3} \mathrm{H}_{5}{ }^{+}$intermediates. Therefore, a discussion for the reaction (4) leading to the formation of both cyclic isomer and linear propargyl cations turns out to be necessary. Fig. 1 represents a schematic reaction coordinate energy profile for the $\mathrm{CH}_{3}{ }^{+}+$ $\mathrm{C}_{2} \mathrm{H}_{2}$ system (Sonnenfroh and Farrar, 1986; Lopez et al., 1996; Almlof et al., 1984; Hariharan et al., 1974). 


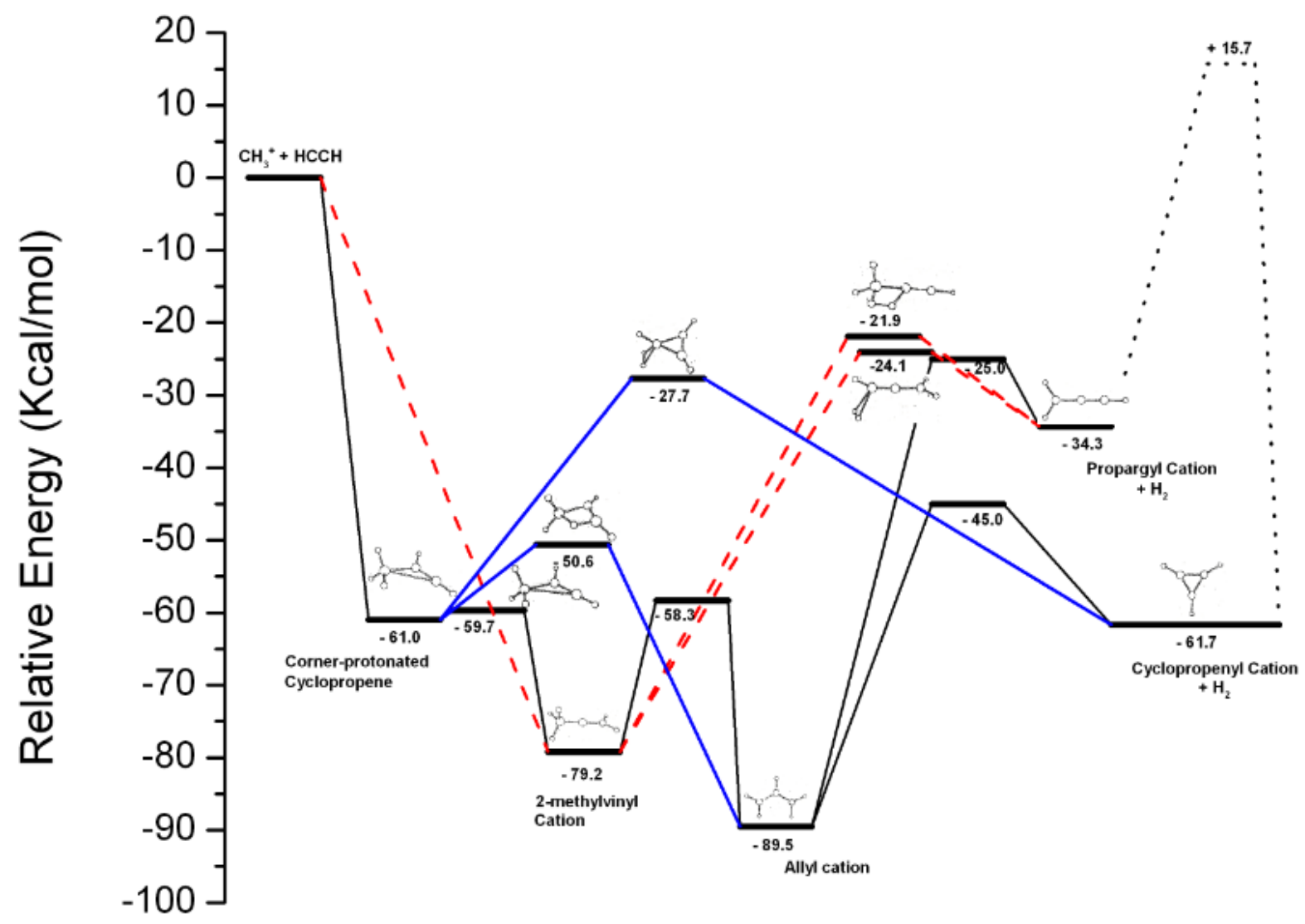

Fig. 1. Schematic potential energy surface for the $\mathrm{CH}_{3}{ }^{+}+\mathrm{C}_{2} \mathrm{H}_{2}$ system. The delocalized Allyl cation is the global minimum. Both products cyclic- $\mathrm{C}_{3} \mathrm{H}_{3}{ }^{+}$and linear- $\mathrm{C}_{3} \mathrm{H}_{3}{ }^{+}$are shown along with the isomerization barrier separating them. The larger circles in structures denote carbon atoms. Three collision complexes and nine transition states are schematically shown in the reaction coordinate plot. The numbers denote zero-point corrected energies (in $\mathrm{Kcal} / \mathrm{mol}$ ) relative to the reactants.

$\mathrm{C}_{3} \mathrm{H}_{3}{ }^{+}$has two isomers: the cyclopropenyl cation and the propargyl cation. The former is approximately $27.4 \mathrm{kcal} / \mathrm{mol}$ more stable than the second one (Lopez et al., 1996). The formation of cyclopropenyl cation $+\mathrm{H}_{2}(\sim-61.7 \mathrm{kcal} / \mathrm{mol})$ as well as the formation of propargyl cation $+\mathrm{H}_{2}(\sim-34.3 \mathrm{kcal} / \mathrm{mol})$ are both exothermic processes in the reaction channel (4) listed above. The reaction of $\mathrm{CH}_{3}{ }^{+}$with acetylene proceeds through the short-lived $\mathrm{C}_{3} \mathrm{H}_{5}{ }^{+}$intermediate which has three different isomeric forms (Lopez et al., 1996): (1) the corner-protonated cyclopropene $(-61.0 \mathrm{kcal} / \mathrm{mol})$ where $\mathrm{CH}_{3}{ }^{+}$is directly above the carbon-carbon double bond forming two-electron three-center bonding, (2) the 2-methylvinyl cation [ $\left.\mathrm{CH}_{3}-\mathrm{C}=\mathrm{CH}_{2}\right]^{+}(-79.2 \mathrm{kcal} / \mathrm{mol})$, and (3) the stable delocalized allyl cation $\left[\mathrm{CH}_{2}=\mathrm{CH}_{-}-\mathrm{CH}_{2}\right]^{+}(-89.5 \mathrm{kcal} / \mathrm{mol})$ the global minimum. Besides these three intermediate collision complexes, recent theoretical calculations (Lopez et al., 1996) have located on the potential energy surface various transition state structures more stable than the reactants of $\mathrm{C}_{3} \mathrm{H}_{5}^{+}$that finally lead to the formation of both isomers of 
$\mathrm{C}_{3} \mathrm{H}_{3}{ }^{+}$. In Fig. 1, the energies shown in brackets are relative to the reactants. The calculations (Lopez et al., 1996) showed that the reactants in channel (4) lead directly to either the corner-protonated cyclopropene or the 2-methylvinyl cation, without encountering any energy barrier. In the entrance channel (see Fig.1), the transition state structure between the corner-protonated cyclopropene and the 2-methylvinyl cation corresponds to an energy barrier of only $1.3 \mathrm{kcal} / \mathrm{mol}$. Similarly, the transition state between the corner-protonated cyclopropene and the allyl cation corresponds to an energy barrier of approximately $10.4 \mathrm{kcal} / \mathrm{mol}$. The transition structure between the most stable intermediate allyl cation and the 2- methylvinyl cation corresponds to an energy barrier of $31.2 \mathrm{kcal} / \mathrm{mol}$. Ab initio calculations (Lopez et al., 1996) also pointed out that both the cyclic- $\mathrm{C}_{3} \mathrm{H}_{3}{ }^{+}$and linear- $\mathrm{C}_{3} \mathrm{H}_{3}{ }^{+}$isomers are generated in the unimolecular decomposition of the collision complex $\mathrm{C}_{3} \mathrm{H}_{5}{ }^{+}$in the bimolecular reaction of $\mathrm{CH}_{3}{ }^{+}$with acetylene. The reaction coordinate calculation (Lopez et al., 1996) identifies in the exit channel three transition state structures: The lowest energy transition state links the corner-protonated cyclopropene to cyclopropenyl cation $+\mathrm{H}_{2}$ products with an energy barrier of $33.3 \mathrm{kcal} / \mathrm{mol}$, with the hydrogen molecule being formed from two $\mathrm{H}$ atoms of the $\mathrm{CH}_{3}{ }^{+}$moiety of the transition state (Fig.1). The other two saddle points (Lopez et al., 1996) lead to the propargyl cation and $\mathrm{H}_{2}$ from the collision complex 2methylvinyl cation. In one of the transition structures the hydrogen molecule is formed from two $\mathrm{H}$ atoms of the $\mathrm{CH}_{3}{ }^{+}$moiety with an energy barrier of $55.1 \mathrm{kcal} / \mathrm{mol}$, while in the other transition state the hydrogen molecule is formed from one $\mathrm{H}$ atom of the $\mathrm{CH}_{3}{ }^{+}$moiety and one $\mathrm{H}$ atom of a $\mathrm{CH}$ group with an energy barrier of $57.3 \mathrm{kcal} / \mathrm{mol}$.

The key question is the isomeric identity of $\mathrm{C}_{3} \mathrm{H}_{3}{ }^{+}$produced from the reaction of $\mathrm{CH}_{3}{ }^{+}$with ethylene and acetylene in Titan's upper atmosphere. As indicated earlier, the cyclic isomer is approximately 27.4 $\mathrm{kcal} / \mathrm{mol}$ more stable than the linear propargyl cation. The intramolecular isomerization leading to product isomers occurs over a barrier of $50 \mathrm{kcal} / \mathrm{mol}$ relative to the propargyl (Fig.1). The total energy available to products in a reaction depends upon reagent internal excitation, relative translational energy and reaction exothermicity. How many $\mathrm{C}_{3} \mathrm{H}_{3}{ }^{+}$isomers are internally excited over their corresponding barrier heights to interconvert? We note that the reaction of $\mathrm{CH}_{3}{ }^{+}$with acetylene is much more exothermic than that with ethylene. The products in the reaction channel (3) are almost isoenergetic (or slightly above in the case of propargyl cation) to the reactants (Sonnenfroh and Farrar, 1986), whereas in channel (4) the products are well below the energy of the reactants (Sonnenfroh and Farrar, 1986; Lopez et al., 1996). The thermochemical limits for propargyl and cyclopropenyl cation formation show that the widths of product center of mass translational energy distributions in the crossed beam measurements (Sonnenfroh and Farrar, 1986) bear on the branching issue between cyclic and linear $\mathrm{C}_{3} \mathrm{H}_{3}{ }^{+}$. With decreasing the relative collision energy of the reagents from $1.15 \mathrm{eV}$ to $0.49 \mathrm{eV}$, the changes in widths of product translational energy distribution for $\mathrm{C}_{3} \mathrm{H}_{3}{ }^{+}$products in the reaction of $\mathrm{CH}_{3}{ }^{+}$with acetylene reflect that the maximum energies available to surmount the transition barrier to propargyl formation decreases substantially (Sonnenfroh and Farrar, 1986). The widths of the kinetic energy distribution at such low collision energies require that a significant fraction (at least 40\%) of products is the most stable cyclic isomer (Sonnenfroh and Farrar, 1986). In the experiment in the relative reagent collision energy from 0.5 to $2.0 \mathrm{eV}$, the lowest kinetic energies for $\mathrm{C}_{3} \mathrm{H}_{3}{ }^{+}$isomers correspond to the most highly internally excited products with excitation above the isomerization 
barriers separating the linear and cyclic isomers. Such products are intrinsically unassignable in the experiment to their isomeric form (Sonnenfroh and Farrar, 1986). The translation energy distributions for the $\mathrm{C}_{3} \mathrm{H}_{3}{ }^{+}$product of the ethylene reaction have been viewed in the same way as those for the acetylene reaction (Sonnenfroh and Farrar, 1986). With decreased reagents relative collision energy in the experiment (Sonnenfroh and Farrar, 1986), the formation of cyclopropenyl cation- the cyclic isomer is more favored.

The temperature at which the various chemical reactions in the gas phase take place in Titan's upper atmosphere is close to $130 \mathrm{~K}$. The temperature could be as low as $10 \mathrm{~K}$ for ion chemistry in interstellar clouds. The initial collision energies in the crossed beam reactive scattering experiments (Sonnenfroh and Farrar, 1986) are much higher by several orders-of-magnitude than the average collision energies at the low temperatures (10-200 K) prevailing in planetary atmospheres and interstellar dense clouds. With respect to temperature dependent kinetic rates and dynamics proceeding over the potential energy surfaces, there are limitations in the current measurements (Sonnenfroh and Farrar, 1986; Burgers et al., 1983). The exact determination of the product branching ratio, e.g., linear $\mathrm{C}_{3} \mathrm{H}_{3}{ }^{+}$versus cyclic $\mathrm{C}_{3} \mathrm{H}_{3}{ }^{+}$ strongly depend upon collision energy. We emphasize the importance of the entrance and exit inner transition state barriers (Sonnenfroh and Farrar, 1986; Lopez et al., 1996) in the reactions of $\mathrm{CH}_{3}{ }^{+}$with ethylene and acetylene mentioned above. In these ion-molecule reactions, in the entrance channel the transition states connecting collision complexes to the covalently bound global minimum all lie well below the reactants (Sonnenfroh and Farrar, 1986; Lopez et al., 1996; Almlof et al., 1984). Thus, simple capture rates (Clary, 1987; Klippenstein and Georgievskii, 2008) in the limit of long range attractive forces can provide an accurate description of the kinetics of the formation of ion-molecule complexes at higher energies around room temperature as well as at the lower collision energies prevailing in planetary atmospheres and interstellar clouds. In treating the unimolecular decomposition of intermediate collision complexes to the products (Wardlaw and Marcus, 1988; Gilbert and Smith, ed. 1990; Holbrook, Pilling, and Robertson, ed. 1996), multiple transition states in the exit channel are involved (Sonnenfroh and Farrar, 1986; Lopez et al., 1996), though they are seldom of kinetic importance. The products in the potential energy surface (Lopez et al., 1996) for the $\mathrm{CH}_{3}{ }^{+}+\mathrm{C}_{2} \mathrm{H}_{2}$ system are linear- $\mathrm{C}_{3} \mathrm{H}_{3}{ }^{+}+\mathrm{H}_{2}$ and Cyclic- $\mathrm{C}_{3} \mathrm{H}_{3}{ }^{+}+\mathrm{H}_{2}$. The exit channel barrier (Lopez et al., 1996) in the potential energy surface for this system connecting the products linear- $\mathrm{C}_{3} \mathrm{H}_{3}{ }^{+}+\mathrm{H}_{2}$ with the 2-methylvinyl cation covalently bound collision complex is approximately $21.9 \mathrm{kcal} / \mathrm{mol}$ below the energy of the reactants, whereas the barrier of the exit saddle point that connects the products cyclic- $\mathrm{C}_{3} \mathrm{H}_{3}{ }^{+}+\mathrm{H}_{2}$ with the cornerprotonated cyclopropene intermediate is approximately $27.7 \mathrm{kcal} / \mathrm{mol}$ below the reactants in energy. We thus expect the formation of both linear and cyclic isomers, with the cyclopropenyl cation as the more favored product (see Fig.1). This is in line with what discussed above, i.e., that the cross beam measurements (Sonnenfroh and Farrar, 1986) of center of mass translational energy distributions for $\mathrm{C}_{3} \mathrm{H}_{3}{ }^{+}$reflect the product branching ratio of cyclic to linear increases with the decrease of reagent relative collision energy from 2.0 to $0.5 \mathrm{eV}$. The dominance of long-range attractive potential (Clary, 1987; Klippenstein and Georgievskii, 2008) in the entrance channel and the multiple transition state models (Lopez et al., 1996) in the product exit channel thus provide clear evidence of the formation of the cyclopropenyl cation, as the major product in the conditions of Titan's upper atmosphere and 
interstellar clouds at low temperatures (10-200 K). The process of isomerization between linear and cyclic isomer of $\mathrm{C}_{3} \mathrm{H}_{3}{ }^{+}$does not occur at temperatures relevant to the chemistry of Titan's atmosphere, because of a very large barrier of about $50 \mathrm{kcal} / \mathrm{mol}$ relative to the linear isomer (Fig.1). The isomerization barrier is higher in energy than that to the reactants.

Similarly, the entrance and exit barriers (Sonnenfroh and Farrar, 1986) in the $\mathrm{CH}_{3}{ }^{+}+\mathrm{C}_{2} \mathrm{H}_{4}$ reaction system determine the product branching ratios at low temperatures (10-200 K). Several transition states have been identified in the exit channel of this reaction. These transition states were shown schematically (Sonnenfroh and Farrar, 1986) and somewhat empirical for three product channels, $\mathrm{C}_{2} \mathrm{H}_{3}{ }^{+}$ $+\mathrm{CH}_{4}, \mathrm{C}_{3} \mathrm{H}_{5}{ }^{+}+\mathrm{H}_{2}$, and $\mathrm{C}_{3} \mathrm{H}_{3}{ }^{+}+2 \mathrm{H}_{2}$. The saddle point in the entrance of the potential energy surface connecting the corner-protonated cyclopropyl cation $\left(\mathrm{C}_{3} \mathrm{H}_{7}{ }^{+}\right)$intermediate (Almlof et al., 1984; Hariharan et al., 1974) to the covalently bound short-lived sec- $\mathrm{C}_{3} \mathrm{H}_{7}{ }^{+}$structure tends to lie (Sonnenfroh and Farrar, 1986) well below the energy of the reactants, because of long-range increased attractions in these ionmolecule complexes. In this respect, simple capture theory (Clary, 1987; Klippenstein and Georgievskii, 2008) provides an accurate description of the long-range part of this attractive potential.

The hydride abstraction reaction or the formation of the product $\mathrm{C}_{2} \mathrm{H}_{3}{ }^{+}+\mathrm{CH}_{4}$ via unimolecular decomposition (Wardlaw and Marcus, 1988; Gilbert and Smith, ed. 1990; Holbrook, Pilling and Robertson, ed. 1996) of sec- $\mathrm{C}_{3} \mathrm{H}_{7}^{+}$in the exit channel does not involve (Sonnenfroh and Farrar, 1986) any transition state barrier. Again, the barrier to the formation of lowest energy allyl cation $\left(\mathrm{C}_{3} \mathrm{H}_{5}^{+}\right)$plus the hydrogen molecule from the complex sec- $\mathrm{C}_{3} \mathrm{H}_{7}{ }^{+}$is approximately $30 \mathrm{kcal} / \mathrm{mol}$ below the energy of the reactants (Sonnenfroh and Farrar, 1986). The isomer products linear- $\mathrm{C}_{3} \mathrm{H}_{3}{ }^{+}+\mathrm{H}_{2}$ are very close in energy or slightly above to that of the reactants. The cyclic $-\mathrm{C}_{3} \mathrm{H}_{3}{ }^{+}+\mathrm{H}_{2}$ are found to be $\sim 20 \mathrm{kcal} / \mathrm{mol}$ below the reactants. There are two large exit channel barriers in the schematic potential energy surface (Sonnenfroh and Farrar, 1986) of sec- $\mathrm{C}_{3} \mathrm{H}_{7}{ }^{+}$for producing both linear and cyclic isomers of $\mathrm{C}_{3} \mathrm{H}_{3}{ }^{+}$from the internally excited intermediate allyl cation $\left(\mathrm{C}_{3} \mathrm{H}_{5}{ }^{+}\right)$. Because the products cyclic $-\mathrm{C}_{3} \mathrm{H}_{3}{ }^{+}+\mathrm{H}_{2}$ are in energy well below the reactants $\mathrm{CH}_{3}{ }^{+}+\mathrm{C}_{2} \mathrm{H}_{4}$, and the exit channel barrier of this reaction does not cross the thermochemical limit, we expect the cyclic isomer is the most dominating form for $\mathrm{C}_{3} \mathrm{H}_{3}{ }^{+}$at relevant low temperatures. Indeed, a very small exit channel barrier does not allow an efficient formation of the linear- $\mathrm{C}_{3} \mathrm{H}_{3}{ }^{+}+\mathrm{H}_{2}$ in the chemistry of atmosphere of Titan and interstellar clouds. To elaborate this specific point and to determine temperature dependent reaction rate coefficients in such complexforming ion-molecule reactions at low temperatures (10-200 K), ab initio electronic structure calculations and microcanonical variational RRKM transition state theory (Wardlaw and Marcus, 1988; Gilbert and Smith, ed. 1990; Holbrook, Pilling, and Robertson, ed.1996) are required in future studies.

Titan's upper atmosphere is chemically complex. The reactions involving neutrals, ion-molecule reactions and the chemistry of negative ions initiate this complexity (Vuitton et al., 2009a, 2009b; Waite et al., 2009; Coates et al., 2007; Sittler et al., 2009; Ali et al., 2010; Klippenstein and Georgievskii, 2008; Balucani, 2009; Larsson et al., 2012) and start the organic inventory in the second largest moon of our solar system. No other planetary environments in our solar system are known to exceed such chemical complexity. The observation of INMS mass spectrum (Vuitton et al., 2009a; Waite et al., 2007) of two coupled ions $\left(\mathrm{C}_{4} \mathrm{H}_{7}{ }^{+}, \mathrm{C}_{4} \mathrm{H}_{5}{ }^{+}\right)$, and $\left(\mathrm{C}_{5} \mathrm{H}_{9}{ }^{+}, \mathrm{C}_{5} \mathrm{H}_{7}{ }^{+}\right)$, and the preceding analysis of the energetics as well as the 
dynamics proceeding over the potential energy surface for the $\mathrm{CH}_{3}{ }^{+}+\mathrm{C}_{2} \mathrm{H}_{2}$ system force us to predict the following "complex-forming" bimolecular reactions of $\mathrm{CH}_{3}{ }^{+}$with methylacetylene and dimethylacetylene in Titan's upper atmosphere:

$$
\begin{aligned}
& \mathrm{CH}_{3}^{+}+\mathrm{HCCCH}_{3} \rightarrow \mathrm{CH}_{3} \mathrm{C}_{3} \mathrm{H}_{2}^{+}+\mathrm{H}_{2}(5) \\
& \mathrm{CH}_{3}{ }^{+}+\mathrm{CH}_{3} \mathrm{CCCH}_{3} \rightarrow \mathrm{CH}_{3} \mathrm{C}_{3} \mathrm{HCH}_{3}{ }^{+}+\mathrm{H}_{2}(6)
\end{aligned}
$$

The reaction (5) is expected to yield molecular hydrogen and the cyclopropenyl cation with one of the hydrogen atoms substituted by a methyl functional group. According to the discussion above, one also expects the formation of the linear isomer- methyl propargyl cation $\left[\mathrm{CH}\left(\mathrm{CH}_{3}\right)=\mathrm{C}=\mathrm{CH}\right]^{+}$in this "condensation reaction" channel. In the reaction (6), the products are the hydrogen molecule and the cyclic isomer of $\mathrm{C}_{3} \mathrm{H}_{3}{ }^{+}$where two hydrogen atoms are replaced by methyl groups. Similarly, the linear isomer $\left[\mathrm{C}\left(\mathrm{CH}_{3}\right)_{2}=\mathrm{C}=\mathrm{CH}\right]^{+}$is also formed in the reaction of $\mathrm{CH}_{3}{ }^{+}$with dimethylacetylene. Both reactions belong to the same condensation reaction class. To the best of our knowledge, the energetics and potential energy characteristics for the ion-molecule reactions (5) and (6) are not known. There are not any laboratory studies of the lifetimes of the collision complexes involved in these two exoergic reactions that can provide the dynamical information and product isomer branching ratios.

Methylacetylene is an abundant molecule (Waite et al., 2009) in Titan's thermosphere and ionosphere. It is a polar molecule and thus we would expect an increased strength in the long-range force (Clary, 1987; Klippenstein and Georgievskii, 2008) in the interaction of a closed-shell ion and a polar molecule in channel (5). Because of this strong long-range interaction, the saddle points connecting ion-molecule complexes to the covalently bound global minimum are expected (Klippenstein and Georgievskii, 2008) to lie well below the reactants in the attractive part of the potential energy surface. We therefore suggest that the simple capture theory (Clary, 1987; Klippenstein and Georgievskii, 2008) is able to provide an accurate description of this ion-dipole reaction kinetics, provided that the potential energy characteristics in the exit channel of the reaction are very similar to that of the $\mathrm{CH}_{3}{ }^{+}+\mathrm{C}_{2} \mathrm{H}_{2}$ system. Similar to the attack of $\mathrm{CH}_{3}{ }^{+}$to acetylene, the ion-molecule reaction (5) forms the $\mathrm{C}_{4} \mathrm{H}_{7}{ }^{+}$collisioncomplex which, via isomeric rearrangement, leads to the global minimum- the delocalized allyl cation (Lopez et al., 1996) where one of the hydrogen atoms in either of the two end carbon atoms is replaced by a methyl group $\left[\mathrm{CH}_{2}=\mathrm{CH}-\mathrm{CH}\left(\mathrm{CH}_{3}\right)\right]^{+}$. In addition to the lowest energy minimum, the $\mathrm{C}_{4} \mathrm{H}_{7}{ }^{+}$complex has two other higher energy isomers: the corner-protonated methylcyclopropene and the 2-methylvinyl cation (Lopez et al., 1996) where one hydrogen of the vinyl cation is replaced by a methyl functional group $\left[\mathrm{CH}_{3}-\mathrm{C}=\mathrm{CH}\left(\mathrm{CH}_{3}\right)\right]^{+}$. Similarly to the reaction of $\mathrm{CH}_{3}{ }^{+}$with acetylene, both higher energy isomers of the collision complex $\mathrm{C}_{4} \mathrm{H}_{7}^{+}$likely evolve to the corresponding products, the cyclic isomer $\mathrm{CH}_{3} \mathrm{C}_{3} \mathrm{H}_{2}{ }^{+}+$ $\mathrm{H}_{2}$ and the linear isomer $\left[\mathrm{CH}\left(\mathrm{CH}_{3}\right)=\mathrm{C}=\mathrm{CH}\right]^{+}+\mathrm{H}_{2}$, through transition state structures. It is the cornerprotonated methylcyclopropene intermediate that is linked to the cyclic isomer of the product in the exit channel of reaction (5), whereas the intermediate structure $\left[\mathrm{CH}_{3}-\mathrm{C}=\mathrm{CH}\left(\mathrm{CH}_{3}\right)\right]^{+}$is connected to the linear product isomer. The lifetime of the intermediate transient complex is only a fraction of the rotational period. As the temperature decreases from room temperature down to $10 \mathrm{~K}$, the decrease of the average collision energy increases the lifetimes of transient complexes (Wardlaw and Marcus, 1988; 
Gilbert and Smith, ed. 1990; Holbrook, Pilling and Robertson, ed. 1996). Because of this increased lifetime, the intermediate complexes in such reactions at low temperatures evolve to the potential global minimum such as $\mathrm{C}_{4} \mathrm{H}_{7}^{+}$and to the methylallyl structure $\left[\mathrm{CH}_{2}=\mathrm{CH}-\mathrm{CH}\left(\mathrm{CH}_{3}\right)\right]^{+}$.

On a similar basis, if we assume that the potential energy characteristics of the system $\mathrm{CH}_{3}{ }^{+}+\mathrm{CH}_{3} \mathrm{CCCH}_{3}$ are similar to that of the $\mathrm{CH}_{3}{ }^{+}+\mathrm{C}_{2} \mathrm{H}_{2}$ system with planar structure of dimethylallyl cation as the global minimum for the intermediate complex $\mathrm{C}_{5} \mathrm{H}_{9}{ }^{+}$, in the reaction channel (6) we expect as well the formation of both products, the cyclic isomer dimethylcyclopropenyl cation $\mathrm{CH}_{3} \mathrm{C}_{3} \mathrm{HCH}_{3}{ }^{+}$and its linear counterpart dimethyl propargyl cation $\left[\mathrm{C}\left(\mathrm{CH}_{3}\right)_{2}=\mathrm{C}=\mathrm{CH}\right]^{+}$.

The key issue we have presented here is the synthesis of the cyclopropenyl cation, the simplest Huckel's aromatic molecule, and its cyclic methyl derivatives. Carbocations observed by the INMS (Waite et al., 2004, 2007, 2009; Vuitton et al., 2009a) play a dominant role in the chemistry of Titan's upper atmosphere. This role starts from the formation of penta- or tetracoordinated "nonclassical" carbonium (Olah, 1972, 1995) collision complexes which, via isomeric rearrangement, lead to the stable $\mathrm{C}_{3} \mathrm{H}_{7}^{+}$, $\mathrm{C}_{3} \mathrm{H}_{5}{ }^{+}, \mathrm{C}_{4} \mathrm{H}_{7}{ }^{+}$, and $\mathrm{C}_{5} \mathrm{H}_{9}{ }^{+}$systems- the global minima on the potential energy surfaces of the reactions involving $\mathrm{CH}_{3}{ }^{+}$with ethylene, acetylene, methylacetylene, and dimethylacetylene, respectively. An understanding of temperature dependent kinetic rate coefficients will ultimately require the microcanonical variational RRKM transition state theory (Wardlaw and Marcus, 1988; Gilbert and Smith, ed. 1990; Holbrook, Pilling, and Robertson, ed. 1996) analysis of both unimolecular decomposition and bimolecular recombination reactions in the $\mathrm{C}_{3} \mathrm{H}_{7}{ }^{+}, \mathrm{C}_{3} \mathrm{H}_{5}{ }^{+}, \mathrm{C}_{4} \mathrm{H}_{7}{ }^{+}$, and $\mathrm{C}_{5} \mathrm{H}_{9}{ }^{+}$systems. It is shown here that the product branching ratios of cyclic to linear isomers of $\mathrm{C}_{3} \mathrm{H}_{3}{ }^{+}$and its methyl derivatives are strongly temperature dependent (10-200 K), and as the temperature of the system decreases down to the temperature of atmospheres of outer planets and their satellites and interstellar clouds, the kinetics of formation of cyclic isomers is dominant. It is further pointed out that in the low temperature limit the kinetics of the reaction of a closed shell ion with a polar or a non-polar molecule in the gas phase can be determined by the capture rate (Klippenstein and Georgievskii, 2008) on the long-range potential, unless the transition state bottlenecks are almost equal in energy or above to the reactants. The simple classical or quantum mechanical capture theory (Clary, 1987; Klippenstein and Georgievskii, 2008) provides a fair description of the chemical reaction rate coefficients at low temperatures of these fast reactions.

\section{Future Studies and Concluding Remarks:}

The detection of a series of carbocations (Waite et al., 2009; Vuitton et al., 2009a) and carbanions (Coates et al., 2007; Vuitton et al., 2009b; Sittler et al., 2009; Ali et al., 2010) in Titan's upper atmosphere by instruments onboard the Cassini mission (Waite et al., 2004; Young et al., 2004) has a very special significance in ion chemistry in space. We reiterate that, among the detected carbocations, $\mathrm{CH}_{5}{ }^{+}$represents the simplest of Olah's (Olah, 1972, 1995) carbocations, and currently this is of considerable interest in space science (Huang et al., 2006; Asvany et al., 2005; McCoy et al., 2004; 
Muller et al., 1997; White et al., 1999). Olah's (Olah, 1972, 1995) three-center two-electron penta- or tetracoordinated "nonclassical" carbonium ions based structure of carbocations is no longer a controversial topic in chemistry. The quantum-mechanical treatment of electron-deficient bonding among three attracting centers of charge was first introduced by Longuet-Higgins, 1957. Many theoretical studies have been published on this subject since that time. Nevertheless, a number of nonequivalent equilibrium structures for $\mathrm{CH}_{5}{ }^{+}$with almost identical energies makes its rotational and vibrational-rotational spectra unusually complicated (White et al., 1999; Bunker et al., 2004). It is perhaps the millimeter-wave laboratory absorption spectroscopy of specific rotational transitions of "sufficiently cold" mass-selected $\mathrm{CH}_{5}{ }^{+}$ion in ion traps (Gerlich, 2005; Ghosh, 1995) that can eventually lead to the astronomical observational confirmation of this fascinating (Olah, 1995) cation, which is predicted to be so abundant in space (Waite et al., 2009; Vuitton et al., 2009a).

The Cassini INMS instrument (Waite et al., 2004) is in essence a quadrupole mass spectrometer. In the upper atmosphere of the moon it measures the mass distributions of a variety of neutrals and positive ions up to 100 amu with one atomic mass unit resolving power between masses (Waite et al., 2005, 2009; Vuitton et al., 2009a). It thus provides primarily information on the chemical compositions. Then, the molecular structures corresponding to the INMS detected composition require spectroscopic features as fingerprints. Thus, there is an urgent need of direct ground-based or in situ spectroscopic observations in the infrared and millimeter-wave region.

Since cyclic- $\mathrm{C}_{3} \mathrm{H}_{3}{ }^{+}$has a $\mathrm{D}_{3 \mathrm{~h}}$ symmetry, it has no permanent dipole moment. Thus, searching this molecule in the atmosphere of Titan by means of pure rotational spectroscopy is not an option. However, various isotopologues of cyclic $-\mathrm{C}_{3} \mathrm{H}_{3}{ }^{+}$via inclusion of one ${ }^{13} \mathrm{C}$ or one $\mathrm{D}$ atom possess a permanent dipole moment (Waite et al., 2005; Abbas et al., 2010). Ab initio quantum-chemical calculations (Huang and Lee, 2011) have recently been performed to compute fundamental vibrational frequencies and spectroscopic constants for rotational and vibro-rotational spectra for the $\mathrm{c}^{13} \mathrm{CC}_{2} \mathrm{H}_{3}{ }^{+}, \mathrm{C}-$ $\mathrm{C}_{3} \mathrm{H}_{2} \mathrm{D}^{+}, \mathrm{C}^{13} \mathrm{CC}_{2} \mathrm{H}_{2} \mathrm{D}^{+}$isotopologues of cyclic- $\mathrm{C}_{3} \mathrm{H}_{3}{ }^{+}$, and the $\mathrm{H}_{2} \mathrm{CCCD}^{+}, \mathrm{HDCCCH}^{+}, \mathrm{H}_{2}{ }^{13} \mathrm{CCCH}^{+}, \mathrm{H}_{2} \mathrm{C}^{13} \mathrm{CCH}^{+}$, and $\mathrm{H}_{2} \mathrm{CC}^{13} \mathrm{CH}^{+}$isotopologues of linear- $\mathrm{C}_{3} \mathrm{H}_{3}{ }^{+}$. The predicted dipole moment values (Huang and Lee, 2011) for the isotopologues of the cyclic isomer noted above are $0.094,0.225$, and $0.312 \mathrm{D}$, respectively, while the linear isotopologues have values that range from 0.325 to $0.811 \mathrm{D}$. Fig.2 depicts the predicted rotational spectra (a-type) for $\mathrm{c}-\mathrm{C}_{3} \mathrm{H}_{2} \mathrm{D}^{+}, \mathrm{C}^{13} \mathrm{CC}_{2} \mathrm{H}_{2} \mathrm{D}^{+}$and $\mathrm{I}-\mathrm{H}_{2} \mathrm{CCCD}^{+}$in the $20-150 \mathrm{GHz}$ frequency range, based on the spectroscopic parameters from Huang and Lee, 2011. We do note that the linear isotopic species, having a dipole moment of $0.811 \mathrm{D}$ and a large rotational constant $\mathrm{A}$, presents a spectrum definitely different from that of the cyclic mono-deuterated isotopologues considered. More interesting is to note that rather different are also the rotational spectra of the two cyclic isotopic species. In fact, despite of having similar dipole moments $(0.225$ and $0.312 \mathrm{D}$ ) and similar rotational parameters (for instance, their $B$ and $C$ values differ by only a few \%), the two rotational spectra are such that allow a clear identification of the two isotopologues. This is well demonstrated by the $J=3_{2,2} \leftarrow 2_{2,1}$ transition shown in the inset of Fig.2: the corresponding frequencies differ by about $3 \mathrm{GHz}$. Due to the large difference in $\mathrm{A}$, for the linear species the same transition lies in a complete different region of spectra. State-of-the-art ab initio quantum-chemical methods have reached such a level that theoretical chemists are able to 
compute very accurate spectroscopic parameters for rotational spectroscopy of small- to medium-sized molecules (Puzzarini et al., 2010). The interplay among theory (Puzzarini et al., 2010), millimeter-

/submillimeter-wave laboratory measurements (Cazzoli and Puzzarini, 2008; Savage and Ziurys, 2005; Cazzoli et al., 2012), and astronomical observations of rotational transitions leads to the most powerful tool to derive molecular structural information for the composition of carbocations (Vuitton et al., 2009a; Waite et al., 2009) determined by Cassini neutral and cationic mass spectrometry (Waite et al., 2004).

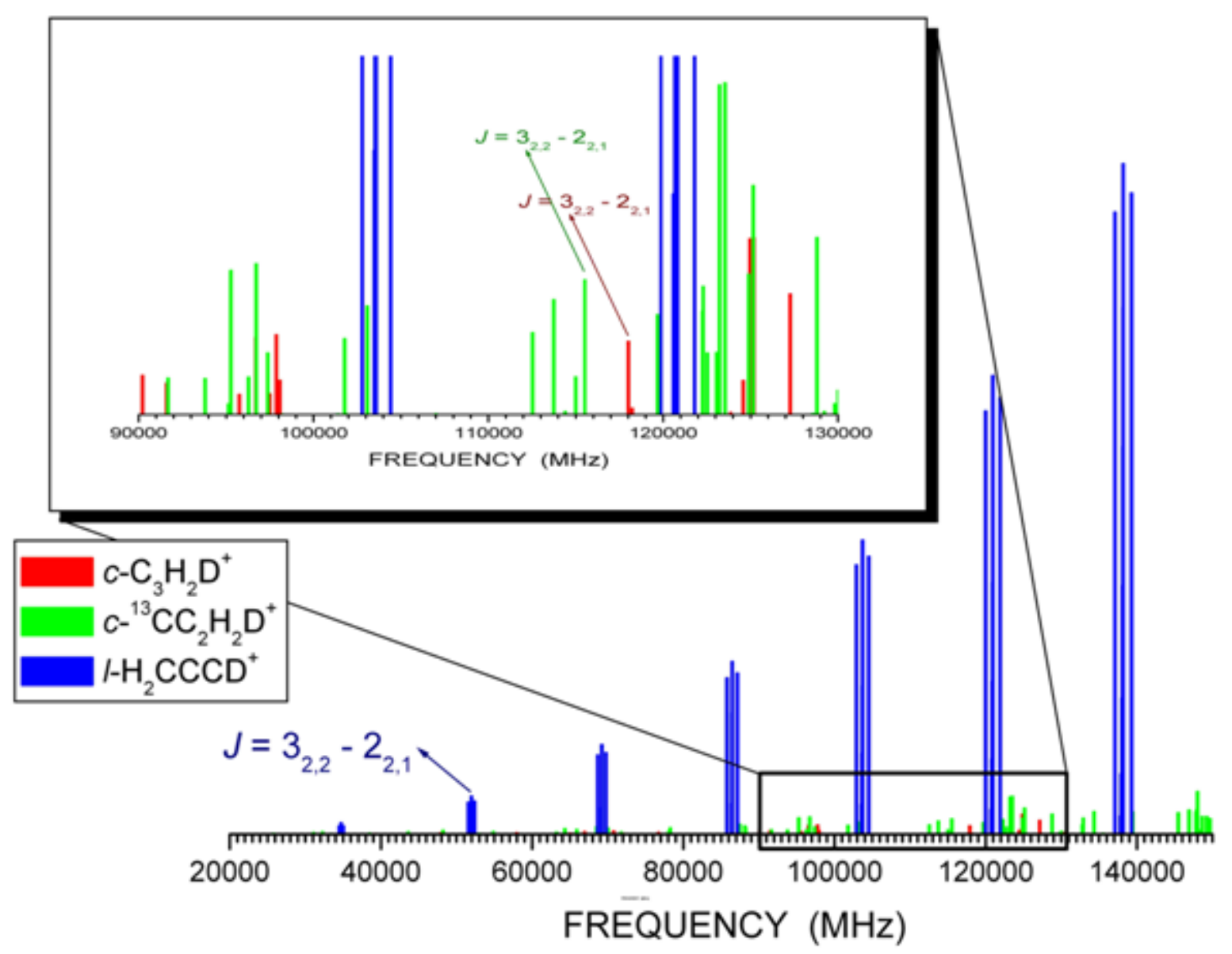

Fig. 2. Calculated pure rotational spectra for ${ }^{13} \mathrm{C}$ and deuterium-containing isotopologues of cyclic and linear $\mathrm{C}_{3} \mathrm{H}_{3}{ }^{+}$in the $20-150 \mathrm{GHz}$ frequency range.

One seminal advance in the field of rotational spectroscopy was the demonstration by Woods and his coworkers that it was possible to observe the rotational spectra of molecular ions at high resolution in a DC discharge plasma (Woods et al., 1975). More recently (Savage and Ziurys, 2005), AC discharges have been used that make it possible to use the velocity modulation (Gudeman and Saykally, 1984; Stephenson and Saykally, 2005) technique (extensively used in the rovibrational spectroscopy) to separate spectral lines due to ions from those arising from neutral species, thus rendering rotational 
spectroscopy (in the millimeter/submillimeter frequency ranges ) well suited for studies of carbocations. Another important advance in the investigation of the rotational spectra of ions was the employment of an axial magnetic field to extend the ion rich negative glow region of an abnormal glow discharge, thus leading to an increase of ion column densities by about a factor of 100 (De Lucia et al., 1983). This approach has been widely adopted for observations of molecular cations in the laboratory, thus making it possible their identification in the interstellar medium. The biggest hurdle (Ricks et al., 2010; Garand et al., 2008) in infrared and microwave spectroscopy experiments is to selectively study a sufficiently cold isomer of a molecular ion in an isolated condition, even if it is well demonstrated that rotational spectrometers based on jet expansion and Fourier Transform technique are able to characterize different conformers at the same time (Cocinero et al., 2012; Pena et al., 2012). As an example, the $\mathrm{C}_{4} \mathrm{H}_{5}{ }^{+}$potential energy surface (Cunje et al., 1996), as evaluated by ab initio computations, is highly complex. A number of minima and several transition structures permitting interconversion between the minima have been located on the potential energy surface. The methylcyclopropenyl cation is the global minimum (Cunje et al., 1996), and five other isomers are above the global minimum by 9.1 to 26.9 $\mathrm{kcal} / \mathrm{mol}$. Because of the existence of multiple minima on the potential energy landscape and substantial barriers to the isomerization processes, each isomer is trapped at the bottom of its corresponding minimum well without the possibility to convert to the lowest energy geometry. As a result, in methylcyclopropenyl cold cation synthetic scheme, the presence of multiple isomers is evident. In an ideal situation, one wants to study infrared or microwave absorption spectra of cold ions (such as $\mathrm{C}_{4} \mathrm{H}_{5}{ }^{+}$) at their lowest energy geometry in rf traps (Garand et al., 2008; Ghosh, 1995). An extension of the absorption spectroscopy technique in molecular structural elucidation is feasible, provided that we find a methodology where the kinetics of the branching ratios of product isomers formation for a given set of reactions can be controlled as the temperature is varied at low temperatures (10-200 K). It is an area where the analysis of spectroscopic properties of equilibrium structures of carbocations requires ab initio quantum-chemical calculations extensively. We expect high-level quantum-chemical methods (Puzzarini, et al., 2010) to be able to compute precisely fundamental vibrational frequencies, rotational constants, vibration-rotation interaction constants and other relevant spectroscopic parameters for the ${ }^{13} \mathrm{C}$ and deuterium-containing isotopologues of the methylcyclopropenyl and dimethylcyclopropenyl cations. Furthermore, unlike the case of charged cyclic- $\mathrm{C}_{3} \mathrm{H}_{3}{ }^{+}$, in both methyl and dimethyl cyclopropenyl cations the center of nuclear charge and the molecular center of mass are no longer the same. It is thus hoped that the laboratory measurements together with the theoretical study of the infrared and millimeter/submillimeter-wave spectra of isotopologues of cyclic- $\mathrm{C}_{3} \mathrm{H}_{3}{ }^{+}$and its methyl derivatives will enable their identification in astronomical observations by means of interferometric arrays such as the Atacama Large Millimeter Array (ALMA), and in the distant future, from a submillimeter-wave heterodyne sounder onboard a Titan orbitter (Lellouch et al., 2010), and from the James Webb Space Telescope (JWST) expected to operate (Gardner et al., 2006) in the near and mid infrared wavelength range between 0.6 and $29 \mu \mathrm{m}$.

Formation of Huckel rings and introduction of aromatic character in cyclic compounds are considered to be the most important classes of reactions in Titan's organic chemistry (Vuitton et al., 2009a; Waite et al., 2009; Sittler et al., 2009). These simplest Huckel's cyclic molecules stabilized by aromaticity play a 
pivotal role in carbocation chemistry in Titan's upper atmosphere. Because of the importance of the chemical pathways for the formation of these aromatics, laboratory and theoretical studies of kinetics and dynamics of specific elementary reactions in the gas-phase such as reactions of $\mathrm{CH}_{3}{ }^{+}$with ethylene, acetylene, methylacetylene and dimethylacetylene at relevant low temperatures (10-200 K) are crucial. Two major advances in experimental techniques (Dupeyrat et al., 1985; Rowe et al., 1984; Smith and Rowe, 2000; Lee, 1987; Balucani, 2009) are now available to characterize the kinetics and dynamics of these elementary reactions in the gas-phase. The first one involves the CRESU (Cinetique de Reaction en Ecoulement Supersonique Uniforme, or Reaction Kinetics in Uniform Supersonic Flow) technique (Rowe et al., 1984; Dupeyrat et al., 1985) that allows the measurements of reaction rate constants at selected low temperatures (10- $200 \mathrm{~K}$ ). Second, the crossed beam study (Lee, 1987; Balucani, 2009) of ionmolecule reactions (Sonnenfroh and Farrar, 1986), especially at very low collision energies, can complement with an important piece of information on the reaction products and their branching ratios. Most importantly, the crossed beam studies probe the reaction dynamics proceeding over potential energy landscape, thus confirming a specific reaction pathway and whether its related products are accessible at low temperatures. Finally, quantum-chemical calculations of energetics and variational RRKM transition state theory (Wardlaw and Marcus, 1988; Gilbert and Smith, ed. 1990; Holbrook, Pilling, and Robertson, ed. 1996) of unimolecular dissociation and bimolecular recombination kinetics of the collision complexes $\mathrm{C}_{3} \mathrm{H}_{7}{ }^{+}, \mathrm{C}_{3} \mathrm{H}_{5}{ }^{+}, \mathrm{C}_{4} \mathrm{H}_{7}{ }^{+}$, and $\mathrm{C}_{5} \mathrm{H}_{9}{ }^{+}$in the reactions mentioned above are needed to provide a mechanistic interpretation of the formation of the simplest form of aromatic cyclic rings in Titan's ionospheric chemistry. As already pointed out, in the low temperature limit the kinetics of gas phase reactions of closed-shell ions with polar and non polar molecules can be well characterized by classical or quantum mechanical simple capture rate (Clary, 1987; Klippenstein and Georgievskii, 2008) on the long-range potential, as the transition states in the entrance channel are well below in energy with respect to the reactants (Sonnenfroh and Farrar, 1986; Lopez et al., 1996).

The formation of complex organic molecules in the interstellar medium proceeds via 'abiotic' synthesis (Klemperer, 2011). The chemistry in Titan's upper atmosphere may tell us a lot about the abiotic model of synthesis of organics in interstellar clouds. The details of processes in Titan's ionospheres differ from ion-molecule reactions in the interstellar medium in a subtle manner (Larsson et al., 2012; Klemperer, 2011). The cyclopropenyl cation $\mathrm{C}_{3} \mathrm{H}_{3}{ }^{+}$has not yet been detected in interstellar clouds. For the formation of $\mathrm{C}_{3} \mathrm{H}_{3}{ }^{+}$, the radiative association reaction of $\mathrm{C}_{3} \mathrm{H}^{+}$with $\mathrm{H}_{2}$ at $10 \mathrm{~K}$ has been proposed (Klemperer, 2011) in interstellar clouds, where $\mathrm{H}_{2}$ is by far the most abundant molecular species. A quantitative understanding of the radiative association rate coefficients (Smith, 1989; Brownsword et al., 1997; Vuitton et al., 2012) is far from being complete. There is an urgent need of an experiment (Gerlich and Horning, 1992) on radiative association rates of $\mathrm{C}_{3} \mathrm{H}^{+}$with $\mathrm{H}_{2}$ at low temperatures, and theory for electronic transition might play a role in this process.

It has been largely accepted that Titan could represent a model of primitive earth (Raulin et al., 2009). The chemistry of carbocations in the nitrogen-methane dominated ionospheres of Titan is immensely important, and this has a possible bearing on organic evolution in the atmosphere of early earth and likely provides the first steps toward a mechanistic theory of aromaticity in terrestrial prebiotic 
chemistry. The radio and infrared observations in the atmospheres of extrasolar planets and in Titan's atmosphere as well should be sought to place a link between organic atmospheric chemistry on Titan and the chemistry in the atmosphere of young earth at its earliest stage of formation in our solar system.

-Formation of terrestrial aromatic cyclic compounds in space is a long standing problem . The long debated Olah's carbocationic chemistry (Olah, 1995; and references therein) has been accepted as a rule rather than exception in physical organic chemistry. In this paper we have identified that an understanding of molecular structures and reactivity of carbocations (detected by Cassini instruments) and their relevance to "nonclassical" carbo-ions will explore aromaticity in the atmosphere of Titan and chemical pathways toward prebiological molecules. Would the chemistry of carbocations be observable in the atmosphere of extrasolar planets? Such astrophysical observational search will most likely place a link between abiotic organic synthetic chemistry in Titan's atmosphere and terrestrial prebiotic chemistry and the origin of life on earth.

The key argument presented in this paper rests on thermochemistry, chemical kinetics and dynamics for the Huckel aromatic structure determination of INMS detected composition (Waite et al., 2004, 2009; Vuitton et al., 2009a) of specific carbocations. To summarize, we have identified an important class of ion-molecule reactions proceeding via carbocation collision complexes, and its implications in chemistry in Titan's upper atmosphere. Based on the kinetics and dynamics of the elementary reactions identified, an analysis of the compositions of ions detected (Vuitton et al., 2009a; Waite et al., 2009) by Cassini cationic mass spectrometry (Waite et al., 2004) reveals the mechanism of formation of the threemembered Huckel aromatic rings -the cyclopropenyl cation and its cyclic methyl derivatives. Emphasis has been given on a synergic effort combining state-of-the-art laboratory experiments, quantum theoretical calculations, and astronomical observations (also including planetary in situ measurements) to elucidate the structure, energetics and dynamics of the compositions of carbocations detected in Titan's thermosphere and ionosphere.

\section{Acknowledgements}

This work was supported in part at NASA Goddard Space Flight Center by the Cassini Plasma Spectrometer (CAPS) Project through NASA Jet Propulsion Laboratory contract 1243218 with the Southwest Research Institute in San Antonio, Texas. C.P. gratefully acknowledges support from Italian MIUR: PRIN 2009 funds (Project: “Molecular Spectroscopy for Atmospherical and Astrochemical Research: Experiment, Theory and Applications"). A.A. thanks Dr. J. Hunter Waite for a critical reading of the manuscript and helpful discussions about the limitations of INMS measurements. We are grateful to both reviewers for a careful reading of the manuscript and for comments. One of the referees (anonymous) summarized the version of our paper. We appreciated his summary so much that we have decided to paraphrase his abstract, and this is included in the last section of the manuscript in a bulleted format. 


\section{References}

Abbas,M.M., Kandadi,H., et al., 2010. D/H Ratio of Titan from Observations of the Cassini/Composite Infrared Spectrometer. Astrophys.J. 708, 342-353.

Ali,A., Chornay,D., Sittler Jr.,E.C., Canosa,A., 2010. Gas-Phase Prebiotic Chemistry on Titan: Specific Laboratory Kinetic Measurements on the Formation Rates and Mechanisms for Molecular Anions Observed by the Cassini Mission. In: Titan Through Time: A Workshop on Titan's Formation, Evolution and Fate. NASA, Goddard Space Flight Center, p. 79.

Almlof,J., Hvistendahl,G., Uggerud,E., 1984. An MC SCF Study of the Reaction $\mathrm{C}_{3} \mathrm{H}_{7}^{+} \rightarrow \mathrm{C}_{3} \mathrm{H}_{5}^{+}+\mathrm{H}_{2}$. Chem.Phys. 90, 55-62.

Amano,T., 2006. Submillimeter-Wave Lines of $\mathrm{H}_{2} \mathrm{D}^{+}$and $\mathrm{D}_{2} \mathrm{H}^{+}$as Probes into Chemistry in Cold Dark Clouds. In: Oka,T. (Eds.), Phil. Trans. R. Soc. A. 364, 2943-2952.

Asvany,O., Kumar,P., Redlich,B., Hegemann, I., Schlemmer,S., Marx,D., 2005. Understanding the Infrared Spectrum of Bare $\mathrm{CH}_{5}{ }^{+}$. Science. 309, 1219-1222.

Balucani,N., 2009. Elementary Reactions and Their Role in Gas-phase Prebiotic Chemistry. Int. J. Mol. Sci. 10, 2304-2335.

Biennier,L., Sabbah,H., Chandrasekaran,V., Klippenstein,S.J., Sims,I.R., Rowe,B.R., 2011. Insights into the Role of Polycyclic Aromatic Hydrocarbon Condensation in Haze Formation in Jupiter's Atmosphere.

Astron.Astrophys. A40, 532-538.

Bogey,M., Cordonnier,M., Demuynck,C., Destombes,J.L., 1992. Laboratory Measurement of the Millimeter and Submillimeter Wave Spectrum of $\mathrm{C}_{2} \mathrm{H}_{3}{ }^{+}$. Astrophys. J. 399, L103-L105.

Boo,D.W., Liu,Z.F., Suits,A.G., Tse,J.S., Lee,Y.T., 1995. Dynamics of Carbonium Ions Solvated by Molecular Hydrgen. Science. 269, 57-59.

Bowman, J.M., Braams,B.J., Carter,S., Chen,C., Czako,G., Fu,B., Huang,X., Kamarchik,E., Sharma, A.R., Shepler,B.C., Wang,Y., Xie,Z., 2010: Ab initio-based Potential Energy Surfaces For Complex Molecules and Molecular Complexes. J. Phys. Chem. Lett. 1, 1866-1874.

Brown,H.C., 1977. The Non-Classical Ion Problem. Plenum, New York.

Brownsword,R.A., Sims,I.R., Smith,I.W.M., Stewart,D.W.A., Canosa,A., Rowe,B.R., 1997. The Radiative Association of $\mathrm{CH}$ With $\mathrm{H}_{2}$ : A Mechanism for Formation of $\mathrm{CH}_{3}$ in Interstellar Clouds. Astrophys. J. 485, 195-202.

Bunker,P.R., Ostojic,B., Yurchenko,S., 2004. A Theoretical Study of the Millimeterwave Spectrum of $\mathrm{CH}_{5}{ }^{+}$ J.Mol.Struc. 695-696, 253-261.

Burgers,P.C., Holmes,J.L., Mommers,A.A., Szulejko,J.E., 1983. The Collisionally Induced Dissociation of Allyl and 2-Propenyl Cations. Org. Mass. Spectrom. 18, 596-600.

Canosa,A., Goulay,F., Sims,I.R., Rowe,B.R., 2008. Gas Phase Reactive Collisions at Very Low Temperatures: Recent Experimental Advances and Perspectives. In: Low Temperatures and Cold Molecules. Smith,I.W.M. (Eds.), Imperial College Press. 55-120

Cazzoli,G., Puzzarini,C., 2008. Lamb-dip Spectrum of Methylacetylene and Methyldiacetylene: Precise Rotational Transition Frequencies and Parameters of the Main Isotopic Species. Astron.Astrophys. 487, 1197-1202.

Cazzoli,G., Cludi,L., Buffa,G., Puzzarini,C., 2012. Precise THz Measurements of $\mathrm{HCO}^{+}, \mathrm{N}_{2} \mathrm{H}^{+}$, and $\mathrm{CF}^{+}$ for Astrophysical Observations. Astrophys. J. Suppl. Series 203, 11.

Clary,D.C., 1988. Theory of Reactive Collisions at Low Temperatures. In: Rate Coefficients in Astrochemistry. Millar,T.J., Williams,D.A. (Eds.), Kluwer Academic Publishers. 1-16.

Coates,A.J., Crary,F.J., Lewis,G.R., Young,D.T., Waite Jr.,J.H., Sittler Jr.,E.C., 2007. Discovery of Heavy Negative lons in Titan's lonosphere. Geophys. Res. Lett. 34, L22103. 
Coates,A.J., Wellbrock,A., Lewis,G.R., Jones,G.H., Young,D.T., Crary,F.J., Waite JR.,J.H., 2009. Heavy Negative lons in Titan's lonosphere: Altitude and Latitude Dependence. Planet. Space Sci. 57, 18661871.

Cocinero,E.J., Lesarri,A., Ecija,P., Basterretxea,F.J., Grabow,J.-U., Fernandez,J.A., Castano,F., 2012. Rotational Spectroscopy: Ribose Found in the Gas Phase. Angew. Chem. Int. Ed. 51, 3119-3124. Crofton,M.W., Jagod,M.F., Rehfuss,B.D., Oka,T., 1989. Infrared Spectroscopy of Carbo-Ions. V. Classical vs Nonclassical Structure of Protonated Acetylene $\mathrm{C}_{2} \mathrm{H}_{3}{ }^{+}$. J. Chem. Phys. 91, 5139-5153. Cunje,A., Rodriquez,C.F., Lien,M.H., Hopkinson,A.C., 1996. The C4H5+ Potential Energy Surface. Structure, Relative Energies, and Enthalpies of Formation of Isomers of $\mathrm{C}_{4} \mathrm{H}_{5}{ }^{+}$. J. Org. Chem. 61, 5212-5220.

De Lucia,F.C., Herbst,E., Plummer,G.M., Blake,G.A., 1983. The Production of Large Concentration of Molecular lons in the Lengthened Negative Glow Region of a Discharge. J.Chem .Phys. 78, 2312-2316.

Drossart,P., Maillard,J.-P., et al., 1989. Detection of $\mathrm{H}_{3}^{+}$on Jupiter. Nature. 340, 539-541

Dupeyrat,G., Marquette,J.B., Rowe,B.R., 1985. Design and Testing of Axisymmetric Nozzles for Ion-Molecule Reaction Studies Between $20^{\circ} \mathrm{K}$ and $160^{\circ} \mathrm{K}$. Phys. Fluids. 28, 1273-1279.

Garand,E., Goebbert,D., Santambrogio,G., Janssens,E., Lievens,P., Meijer,G., Neumark, D.M., Asmis,K.R., 2008. Vibrational Spectra of Small Silicon Monoxide Cluster Cations Measured by Infrared Multiple Photon Dissociation Spectroscopy. Phys. Chem. Chem. Phys. 10, 1502-1506.

Gardner,J.P., Mather,J.C., et al., 2006. The James Webb Space Telescope. Space. Sci. Rev. 123, 485-606.

Gerlich,D., 2005. Probing the Structure of $\mathrm{CH}_{5}{ }^{+}$Ions and Deuterated Variants Via Collisions.

Phys. Chem. Chem. Phys. 7, 1583-1591.

Gerlich,D., Horning,S., 1992. Experimental Investigations of Radiative Association Processes as Related to Interstellar Chemistry. Chem. Rev. 92, 1509-1539.

Ghosh,P.K., 1995. Ion Traps. Clarendon Press, Oxford.

Gilbert,R.G., Smith,S.C., 1990. Theory of Unimolecular and Recombination Reactions. Blackwell Scientific Boston.

Gudeman,C.S., Saykally,R.J., 1984. Velocity Modulation Infrared Laser Spectroscopy of Molecular lons. Ann. Rev. Phys. Chem. 35, 387-418.

Hariharan,P.C., Radom,L., Pople,J.A., Schleyer,P.v.R., 1974. Molecular Orbital Theory of the Electronic Structure of Organic Compounds. XX. $\mathrm{C}_{3} \mathrm{H}_{7}{ }^{+}$Cations with a Polarized Basis Set. J. Amer. Chem. Soc. 96, 599-601.

Herbst,E., 2005. Chemistry of Star-Forming Regions. J. Phys. Chem. A. 109, 4017-4029.

Hinkle,C.E., Petit,A.S., McCoy,A.B., 2011: Diffusion Monte Carlo studies of low energy ro-vibrational states of and its deuterated isotopologues. J. Mol. Spectrosc. 268, 189-198.

Hoffmann,R., 1964. Extended Huckel Theory. IV. Carbonium Ions. J. Chem. Phys. 40, 2480-2488. Holbrook,K.A., Pilling,M.J., Robertson,S.H., 1996. Unimolecular Reactions (Ed.2.). Wiley, New York. Huang,X., Lee,T. J., 2011. Spectroscopic Constants for ${ }^{13} \mathrm{C}$ and Deuterium Isotopologues of Cyclic and Linear $\mathrm{C}_{3} \mathrm{H}_{3}^{+}$. Astrophys.J. 736:33, 1-9.

Huang,X., McCoy,A.B., Bowman,J.M., Johnson,L.M., Savage,C., Dong,F., Nesbitt,D.J., 2006. Quantum Deconstruction of the Infrared Spectrum of $\mathrm{CH}_{5}{ }^{+}$. Science. 311, 60-63.

Ivanov, S.D., Asvany,O., Witt,A., Hugo,E., Mathias,G., Redlich,B., Marx,D., Schlemmer,S., 2010: Quantuminduced symmetry breaking explains infrared spectra of $\mathrm{CH}_{5}{ }^{+}$isotopologues. Nature Chem. 2, 298-302.

Klemperer,W., 2011. Astronomical Chemistry. Annu. Rev. Phys. Chem. 62, 173-184.

Klippenstein,S.J., Georgievskii,Y., 2008. Theory of Low Temperature Gas-Phase Reactions. In: Low Temperatures and Cold Molecules. Smith,I.W.M., (Eds.), Imperial College Press. 175-229.

Larsson,M., Geppert,W.D., Nyman,G., 2012. Ion Chemistry in Space. Rep.Prog. Phys. 75, 066901(75pp). Lavvas,P., Yelle,R.V., et al., 2013. Aerosol Growth in Titan's lonosphere. PNAS. 110, 2729-2734. 
Lee,Y.T., 1987. Molecular Beam Studies of Elementary Chemical Processes. Science. 236, 793-798.

Lellouch,E., Vinatier,S., Moreno,R., Allen,M., Gulkis,S., Hartogh,P., Krieg,J.-M., Maestrini,A., Mehdi,I., Coustenis,A., 2010. Sounding of Titan's Atmosphere at Submillimeter Wavelengths from an Orbiting Spacecraft. Planet. Space Sci. 58, 1724-1739.

Lindh,R., Rice,J.E., Lee,T.J., 1991. The Energy Separation between the Classical and Nonclassical Isomers of Protonated Acetylene. An Extensive Study in One- and n-Particle Space Saturation. J. Chem. Phys. $94,8008-8014$.

Longuet-Higgins,H.C., 1957. The Structures of Electron-Deficient Molecules. Quart. Rev. 11, 121-133. Lopez,R., Sordo,J.A., Sordo,T.L., Schleyer,P.V.R., 1996. Ab Initio Study of the Formation of $\mathrm{C}_{3} \mathrm{H}_{3}{ }^{+}$from The Reaction of $\mathrm{CH}_{3}{ }^{+}$with Acetylene. J. Comput. Chem. 17, 905-909.

Marx,D., Savin,A., 1997. Topological Bifurcation Analysis: Electronic Structure of $\mathrm{CH}_{5}{ }^{+}$. Angew. Chem. Int. Ed. Engl. 36, 2077-2080.

McCoy,A.B., Braams,B.J., Brown,A., Huang,X., Jin,Z., Bowman,J.M., 2004. Ab Initio Diffusion Monte Carlo Calculations of the Quantum Behavior of $\mathrm{CH}_{5}{ }^{+}$in Full Dimensionality. J. Phys. Chem. A. 108, 4991-4994. McGuire,B.A., Wang,Y., Bowman,J.M., Widicus Weaver,S.L., 2011, Do $\mathrm{H}_{5}{ }^{+}$and Its Isotopologues Have Rotational Spectra? J. Phys. Chem. Lett., 2, 1405-1407

Michael,M., Tripathi,S.N., Arya,P., Coates,A.J., Wellbrock,A., Young,D.T., 2011. High-Altitude Charged Aerosols in the Atmosphere of Titan. Planet. Space Sci. 59, 880-885.

Muller,H., Kutzelnigg,W., Noga,J., Klopper,W., 1997. $\mathrm{CH}_{5}{ }^{+}$: The Story Goes On. An Explicitly Correlated Coupled-Cluster Study. J. Chem. Phys. 106, 1863-1869.

Niemann,H.B., Atreya,S.K., et al., 2002. The Gas Chromatograph Mass Spectrometer for the Huygens Probe. Space Sci. Rev. 104, 553-591.

Oka,T., 2006. Interstellar H3+. Proc. Nat. Acad. Sci. 103, 12235-12242; and references therein.

Oka,T., 2012. Physics, Chemistry, and Astronomy of $\mathrm{H}_{3}{ }^{+}$. (Eds.). Phil. Trans. R. Soc. A. Royal Society Discussion Meeting.

Olah, G.A., 1972. The General Concept and Structure of Carbocations Based on Differentiation of Trivalent ("Classical") Carbenium Ions from Three-Center Bound Penta- or Tetracoordinated ("Nonclassical") Carbonium Ions. The Role of Carbocations in Electrophilic Reactions.

J. Amer. Chem. Soc. 94, 808-820.

Olah,G.A., 1995. My Search for Carbocations and Their Role in Chemistry (Nobel Lecture). Angew. Chem. Int. Ed. Engl. 34, 1393-1405.

Park,K., Light,J.C., 2007. Microcanonical Statistical Study of Ortho-Para Conversion in the Reaction $\mathrm{H}_{3}{ }^{+}+\mathrm{H}_{2} \rightarrow\left(\mathrm{H}_{5}{ }^{+}\right)^{*} \rightarrow \mathrm{H}_{3}{ }^{+}+\mathrm{H}_{2}$ at Very Low Energies. J. Chem. Phys. 126, 044305 (1-19).

Pena,I., Sanz,M.E., Lopez,J.C., Alonso.,J.L., 2012. Preferred Conformers of Proteinogenic Glutamic Acid. J. Amer. Chem. Soc. 134, 2305-2312.

Puzzarini,C., Stanton,J.F., Gauss,J., 2010. Quantum-Chemical Calculation of Spectroscopic Parameters for Rotational Spectroscopy. Int. Rev. Phys. Chem. 29, 273-367.

Raulin,F., McKay,C., Lunine,J., Owen,T., 2009. Titan's Astrobiology. In: Brown,R.H., et al. (Eds.), Titan from Cassini-Huygens. Springer, pp. 215-233.

Ricks,A.M., Douberly,G.E., Schleyer,P.v.R., Duncan,M.A., 2010. Communications: Infrared Spectroscopy of Gas Phase $\mathrm{C}_{3} \mathrm{H}_{3}{ }^{+}$Ions: The Cyclopropenyl and Propargyl Cations. J. Chem. Phys. 132, 051101 (1-4).

Rowe,B.R., Dupeyrat,G., Marquette,J.B., Gaucherel,P., 1984. Study of the Reactions $\mathrm{N}_{2}^{+}+2 \mathrm{~N}_{2} \rightarrow \mathrm{N}_{4}{ }^{+}+\mathrm{N}_{2}$ and $\mathrm{O}_{2}{ }^{+}+2 \mathrm{O}_{2} \rightarrow \mathrm{O}_{4}{ }^{+}+\mathrm{O}_{2}$ from 20 to $160 \mathrm{~K}$ by the CRESU technique. J. Chem. Phys. 80, 4915-4921.

Savage,C., Ziurys,L.M., 2005. A Millimeter/Submillimeter Velocity Modulation Spectrometer for Studies of Molecular lons. Rev. Sci. Instrum. 76, 043106 (1-6).

Saykally,R.J., 1988. Infrared Laser Spectroscopy of Molecular lons. Science. 239, 157-161.

Schreiner,P.R., Kim,S.-J., Schaefer,H.F., Schleyer,P.v.R., 1993. $\mathrm{CH}_{5}{ }^{+}$: The Never-Ending Story or the Final 
Word? J. Chem. Phys. 99, 3716-3720.

Sittler Jr.,E.C., Ali,A., Cooper,J.F., Hartle,R.E., Johnson,R.E., Coates,A.J., Young,D.T. 2009. Heavy lon Formation in Titan's lonosphere: Magnetospheric Introduction of Free Oxygen and a Source of Titan's Aerosols ? Planet. Space Sci. 57, 1547-1557.

Smith,I.W.M., 1989. Effects of Quantum Mechanical Tunneling on Rates of Radiative Association. Astrophys. J. 347, 282-288.

Smith,I.W.M., Rowe,B.R., 2000. Reaction Kinetics at Very Low Temperatures: laboratory Studies and Interstellar Chemistry. Acc. Chem. Res. 33, 261-268.

Sonnenfroh,D.M., Farrar,J.M.,1986. Collision Complexes in the Reactions of $\mathrm{CH}_{3}{ }^{+}$with $\mathrm{C}_{2} \mathrm{H}_{4}$ and $\mathrm{C}_{2} \mathrm{H}_{2}$. J.Chem.Phys. 85, 7167-7177.

Stephenson,S.K., Saykally,R.J., 2005. Velocity Modulation Spectroscopy of Ions. Chem. Rev. 105, 3220-3234.

Vuitton,V., Yelle,R.V., Lavvas,P., Klippenstein,S.J., 2012. Rapid Association Reactions at Low Pressure: Impact on the Formation of Hydrocarbons on Titan. Astrophys.J. 744:11 (7pp).

Vuitton,V., Yelle,R.V., Lavvas,P., 2009a. Composition and Chemistry of Titan's Thermosphere and Ionosphere. Phil. Trans. R. Soc. A. 367, 729-741.

Vuitton,V., Lavvas,P., Yelle,R.V., Galand,M., Wellbrock,A., Lewis, G.R., Coates,A.J., Wahlund,J.-E., 2009b. Negative Ion Chemistry in Titan's Upper Atmosphere. Planet. Space Sci. 57, 1558-1572.

Waite,Jr.,J.H., Young,D.T., Westlake,J.H., Lunine,J.I., McKay,C.P., Lewis,W.S., 2009. High-Altitude Production of Titan's Aerosols. In: Brown,R.H., et al. (Eds.), Titan from Cassini-Huygens. Springer, pp. 201-214.

Waite,Jr.,J.H., Niemann,H.B., et al., 2005. Ion Neutral Mass Spectrometer Results from the First Flyby of Titan. Science. 308, 982-986.

Waite,Jr.,J.H., Lewis,W.S., et al., 2004. The Cassini Ion and Neutral Mass Spectrometer (INMS) Investigation. Space Sci. Rev. 114, 113-231.

Waite,Jr.,J.H., Young,D.T., Cravens,T.E., Coates,A.J., Crary,F.J., Magee,B., Westlake,J., 2007. The Process of Tholin Formation in Titan's Upper Atmosphere. Science. 316, 870-875.

Wardlaw,D.M., Marcus,R.A., 1988. On the Statistical Theory of Unimolecular Processes.

Adv. Chem. Phys. 70, part2, 231-263.

White,E.T., Tang,J., Oka,T., 1999. $\mathrm{CH}_{5}{ }^{+}$: The Infrared Spectrum Observed. Science. 284, 135-137.

Witt,A., Ivanov,S.D., Mathias,G., Marx,D., 2011: Quantum Molecular Dynamics Calculations of Ultrafast Time Scales and Infrared Spectra of Protonated Methane: Quantifying Isotope-Specific Lifetimes. J. Phys. Chem. Lett. 2, 1377-1381.

Woods,R.C., Dixon,T.A., Saykally,R.J., Szanto,P.G., 1975. Laboratory Microwave Spectrum of $\mathrm{HCO}^{+}$. Phys. Rev. Lett. 35, 1269-1272.

Young,D.T., Berthelier,J.J., et al., 2004. Cassini Plasma Spectrometer Investigation. Space Sci. Rev. 114, 1-112. 
FIG.1. Schematic potential energy surface for the $\mathrm{CH}_{3}{ }^{+}+\mathrm{C}_{2} \mathrm{H}_{2}$ system. The delocalized Allyl cation is the global minimum. Both products cyclic- $\mathrm{C}_{3} \mathrm{H}_{3}{ }^{+}$and linear- $\mathrm{C}_{3} \mathrm{H}_{3}{ }^{+}$are shown along with the isomerization barrier separating them. The larger circles in structures denote carbon atoms. Three collision complexes and nine transition states are schematically shown in the reaction coordinate plot. The numbers denote zero-point corrected energies (in $\mathrm{Kcal} / \mathrm{mol}$ ) relative to the reactants.

FIG.2. Calculated pure rotational spectra for ${ }^{13} \mathrm{C}$ and deuterium-containing isotopologues of cyclic and linear $\mathrm{C}_{3} \mathrm{H}_{3}{ }^{+}$in the $20-150 \mathrm{GHz}$ frequency range. 